\title{
MedienPädagogik
}

www. medienpaed.com

Zeitschrift für

Theorie und Praxis

der Medienbildung

ISSN 1424-3636

Themenheft Nr. 23: Visuelle Kompetenz

\section{Visuelle Narreme und die Ästhetisierung des Politischen - Erinnerungskultur 2.0}

Hermann Mitterhofer

\begin{abstract}
Der Beitrag widmet sich der Analyse einer Webseite auf SPIEGEL-Online, die die Terroranschläge auf das World Trade Center am 11. September 2001 im Zuge des Erinnerns zehn Jahre später rekonstruiert. Ausgehend von einer Beschreibung der Diskursfragmente, die sich aus verschiedenen digitalen Archiven speisen, wird der Frage nachgegangen, um welche Art von Erinnerungsort es sich handelt. Vor diesem Hintergrund wird die diskursive Struktur der Webseite einer Analyse unterzogen. Diese Analyse bildet den Ausgangspunkt einer Auseinandersetzung mit der Bedeutung der Diskursfragmente, insbesondere der Semantik der visuellen Elemente. Im Zentrum wird zunächst die Frage der inneren Struktur der Webseite stehen, die Anordnung der Bilder, die, so die These, einer narrativen Logik folgt. Im Weiteren wird der Frage nachgegangen, welche Rolle die Ästhetisierung als Bedingung dafür spielt, dass bestimmte Pressefotografien zu politischen Ikonen gerinnen. Ein möglicherweise überraschender Effekt, der aus dem Spannungsfeld «Schlagbild» (Aby Warburg), Ästhetisierung und emotionaler Erinnerung erwachsen kann, vervollständigt den Beitrag.
\end{abstract}

"Asche, Feuer, Todesangst» - diese Worte sind auf einer Webseite zu lesen, die den Titel trägt «Was am Tag des Terrors geschah: 9/11 in Bildern und Tönen». Zu sehen sind die beiden Türme des World Trade Centers, die weit über die umliegenden Gebäude nach oben ragen. Farblich vorherrschend ist grau und beige, die Häuser am oberen Bildrand verlieren sich in einem stilisierten Mix aus Dunst und Nebel, rechts im Bild ist der Ausläufer der Brooklyn-Bridge über dem East River zu sehen. Entlang der graphisch gestalteten Zwillingstürme sind Video- und AudioSymbole angebracht. Der Text verrät: «Diese Bilder und Töne gingen um die Welt. Die Anschläge vom 11. September 2001 wurden in spektakulären Video- und Audioaufnahmen festgehalten - und haben bis heute nichts von ihrem Schrecken verloren.» Und fett hervorgehoben: «Für einen chronologischen Ablauf klicken Sie auf die Zeitleiste am unteren Bildrand.» Klickt man auf die Symbole erscheinen kleinere Fenster: Zu sehen ist beispielsweise das Porträtfoto einer Stewardess aus dem ersten entführten Flugzeug und gleichzeitig ist der Telefonmitschnitt ihres Notrufs zu hören; Standbilder der Explosion im Südturm nach dem Einschlag des zweiten

https://doi.org/10.21240/mpaed/23/2013.06.25.X

Online publiziert: 25. Juni 2013 
Flugzeugs sind zu sehen, verknüpft mit Tonaufzeichnungen der Einsatzkräfte, die das Geschehen kommentieren; zu hören ist ein Notruf aus dem Südturm, die Stimme des Mannes reisst jäh im Satz ab und zwar in exakt dem Moment, als der Turm einstürzt - mittels Montage visuell effektvoll in Szene gesetzt; und nicht zuletzt ist der Einsturz des Nordturms zu sehen, es sind die Bilder der kaskadenartig herabstürzenden Trümmer und der sich ausbreitenden Aschewolke.

Diese im eigentlichen Sinne interaktive Infographik erschien zehn Jahre nach den Terroranschlägen in New York im Kontext des Erinnerns auf SPIEGEL-Online:

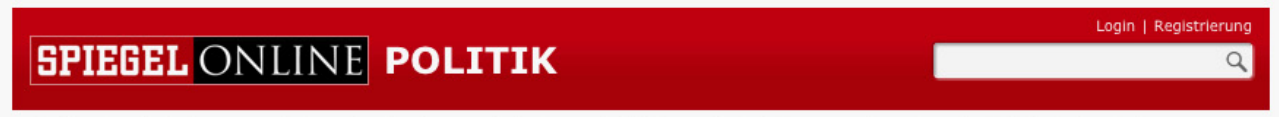

Politik Wirtschaft Panorama $\mid$ Sport Kultur Netzwelt Wissenschaft Gesundheit einestages Karriere Uni Schule Reise Auto

Nachrichten $>$ Polltik $>11$. September 2001

8

11. September 2001

Alle Artikel und Hintergründe

Was am Tag des Terrors geschah: 9/11 in Bildern und Tönen

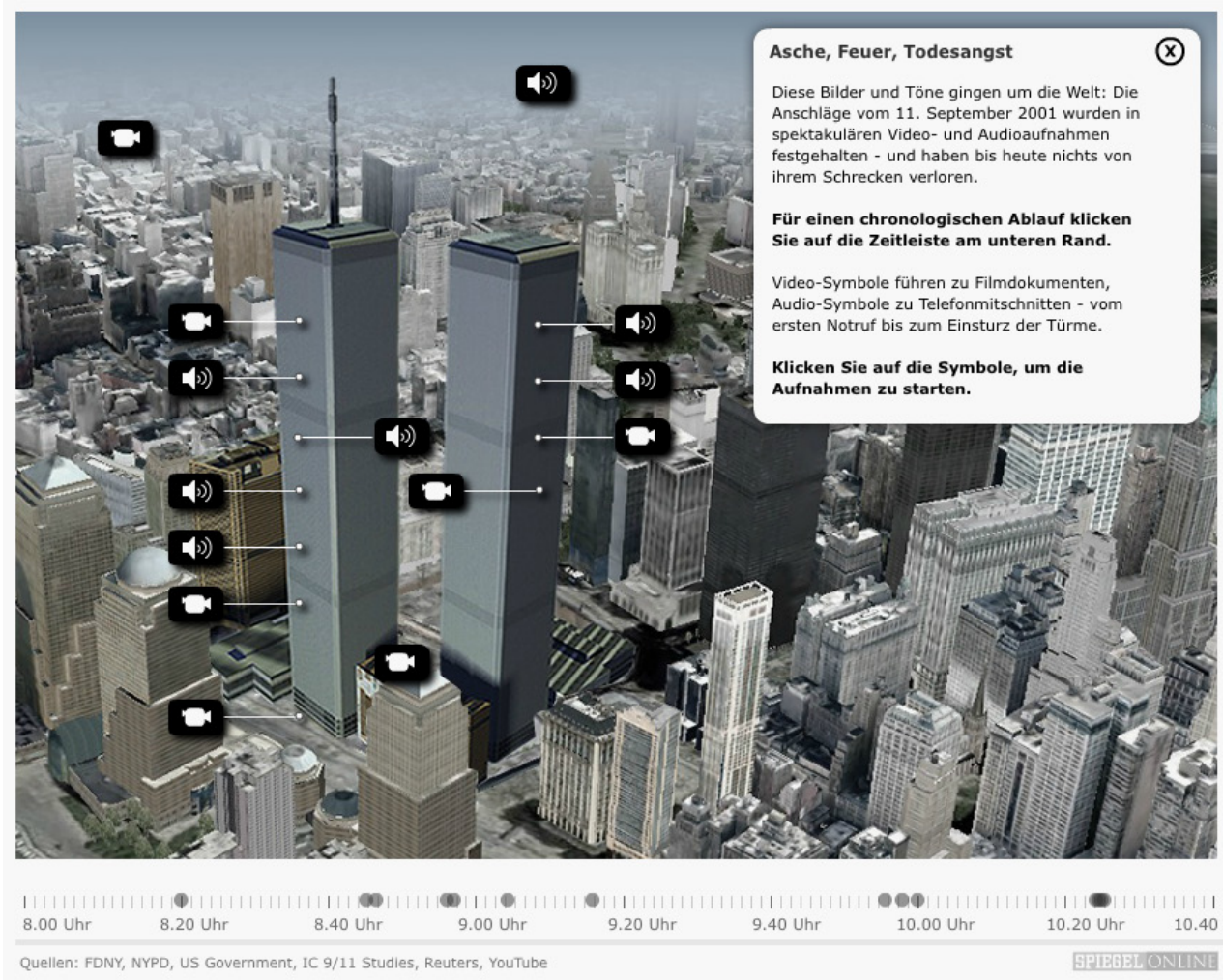


Die einzelnen Diskursfragmente (Jäger 2004, S. 159) entstammen digitalen Archiven wie beispielsweise dem des Fire Departments New York, des New York Police Departments, des International Center for 9/11 Studies und des United States District Courts - Eastern District of Virginia. Bei Letzterem handelt es sich um die Veröffentlichung von Beweismitteln im Zuge eines Gerichtsverfahrens: Erstmals in der Geschichte des US-Justizwesens wurden im Jahre 2006 nach der Verurteilung eines Angeklagten, Zacarias Moussaoui, im Kontext der Terroranschläge von 2001 beinahe alle Beweisstücke dieses Prozesses - sowohl der Anklage wie der Verteidigung - einer breiteren Öffentlichkeit zugänglich gemacht. Es handelt sich um insgesamt 1202 Dokumente, die im Internet veröffentlicht wurden, nur sieben Beweisstücke wurden zurückgehalten. ${ }^{1}$

In diesem Beitrag wird zunächst der Frage nachgegangen, welche Rolle dem Internet als digitalem Ort kollektiver Erinnerung zukommt. Davon ausgehend wird die SPIEGEL-Webseite exemplarisch als möglicher inszenierter Erinnerungsort einer Analyse unterzogen. Zwei Fragen stehen im Zentrum der Auseinandersetzung: Zum einen, welche innere Struktur weist die Webseite auf? - Damit meine ich nicht die technische, programmierte Seite, sondern die diskursiven Tiefenstrukturen. Der Begriff des Diskurses wird hier im Sinne Michel Foucaults als Gesamtheit von Praktiken verstanden, "die systematisch die Gegenstände bilden, von denen sie sprechen» (Foucault 1990, S. 74). Zum anderen wird nach der Bedeutung des Visuellen gefragt, genauer, nach den Eigenschaften so genannter Medienikonen, um die es sich bei den Bildern aus New York zweifelsfrei handelt, den Effekten, die von ihnen ausgehen und dem spezifischen Charakter, den sie auf der Webseite annehmen.

\section{Globital Memory Field und die Struktur der Narreme}

Die SPIEGEL-Webseite, so die erste These, präsentiert sich als Erinnerungsort des kommunikativen Gedächtnisses (Assmann 2007, S. 50). In modernen Gesellschaften übernehmen audiovisuelle Medien (Meyer 2009b, S. 175) und das World Wide Web zunehmend die Aufgabe, an (zeit-)historische Ereignisse zu erinnern. Mit Blick auf das Internet wird von einer Erinnerungskultur 2.0 gesprochen: Digitale Medien, die Texte wie Bilder, insbesondere Fotografien verbreiten, beeinflussen zunehmend unsere Sicht auf Vergangenheit und «begründen neue Formen von Geschichtsvermittlung und Opfergedenken» (Meyer 2009a, S. 7). Anna Reading weist auf den Umstand hin, dass diese Verknüpfung von Neuen Medien und Gedächtnis auch vor dem Hintergrund der Dynamik der Globalisierung zu sehen ist: Zunehmende Globalisierung und Digitalisierung generieren ein globital memory field (Reading 2011, S. 241 f.). Dieser Neologismus führt «global» und «digital»

1 http://www.spiegel.de/panorama/justiz/moussaoui-beweise-online-terrorpuzzle-mit-1200-teilena-429858.html. 
mit Erinnerungskulturen und Pierre Bourdieus «Feld»-Konzept zusammen. Es stellt sich demnach die Frage, welche innere Struktur die SPIEGEL-Webseite aufweist, durch die sie als globale Kommemoration an 9/11 dient.

Im Folgenden möchte ich zeigen, dass dieser Webseite eine spezifische Struktur zugrunde liegt: die Struktur einer Erzählung. Anders formuliert, die einzelnen diskursiven Bausteine, die Diskursfragmente - insbesondere die Bilder - sind so angeordnet, dass sie der Logik einer Narration folgen. In Anlehnung an den Anglisten Werner Wolf möchte ich als Ausgangsposition die Meinung vertreten, dass Erzählen «als Akt des Hervorbringens von Geschichten [...] weit über das Medium Literatur und verbale Textsorten hinaus [geht]: Erzählen ist intermedial» (Wolf 2002, S. 23). Erzählen schliesst damit Diskursfragmente wie Töne und Bilder explizit mit ein. Das Narrative selbst ist eine Struktur mit relativer Konstanz, die meist auf lebensweltlicher Erfahrung aufbaut und nach Mieke Bal (vgl. Wolf 2002, S. 34) zumindest die beiden Grundelemente Zeitlichkeit und Ereignishaftigkeit enthalten muss, ohne dass eine Festlegung bestimmter Realisierungs- oder Vermittlungsmedien erforderlich ist. Ähnlich verhält es sich mit dem Deskriptiven oder Argumentativen, beides sind strukturierende Schemata (Wolf 2002, S. 37). Das Narrative ist gekennzeichnet durch die Qualität der Narrativität. Narrativität ist ein formaler Faktor, inhaltsunspezifisch (Wolf 2002, S. 38): Ein und dieselbe Geschichte, in der strukturalistischen Narratologie histoire (Tzvetan Todorov) oder story (Seymour Chatman) genannt, kann in verschiedenen Medien in Erscheinung treten, in mündlicher, schriftlicher oder fotografischer Form. Die Narrativität ist somit ein Strukturmodell zur graduierbaren Festlegung des Narrativen bzw. Erzählerischen eines Werks. Die Faktoren, die den Grad an Narrativität einer Geschichte feststellen lassen, werden im Anschluss an Wolf Narreme genannt (ebd.). Auch sie sind nicht auf verbale oder schriftliche Ausdrucksformen beschränkt. Narreme stehen im Folgenden im Mittelpunkt, um die These zu erhärten, dass sich die SPIEGEL-Webseite an einer narrativen Logik orientiert und sich als interaktive, digitale, narrative Vermittlungsform - im strukturalistischen Paradigma discourse ${ }^{2}$ genannt - präsentiert. Narreme gliedern sich nach Wolf in drei Gruppen: (1) qualitative Narreme, sie orientieren sich an allgemeinen Kennzeichen des Narrativen; (2) inhaltliche Narreme, Wolf nennt sie «Hohlformen» oder building blocks (Marie-Laure Ryan); (3) syntaktische Narreme, sie kennzeichnen syntaktische Regeln oder Prinzipien für deren Selektion, Verbindung und Präsentation (Wolf 2002, S. 44).

Zu den Basisfunktionen des Narrativen - und damit zu (1), den qualitativen Narremen - zählt man bestimmte Grundqualitäten, die Erzählen kennzeichnen: eine die Zeitebene einbeziehende Sinndimension, eine Darstellungsqualität, die sich aus der repräsentierenden und (re-)konstruierenden Funktion ergibt und eine Erlebnis-

2 Der Begriff des discourse in der strukturalen Erzähltheorie ist nicht gleichzusetzen mit dem Diskurs-Begriff von Michel Foucault wie er oben eingeführt wurde. 
qualität, die sich aus der kommunikativen Funktion ableitet (Wolf 2002, S. 44). Die SPIEGEL-Webseite erfüllt alle drei Kriterien: Es wird eine «sinnvolle», kohärente Welt dargestellt, New York im September 2001 und ein Ereignis re-konstruiert. Die Erlebnisqualität ist durch die interaktive Lösung, die frei wählbare Auswahl der Fenster und durch die Wiederholbarkeit gegeben.

Inhaltliche Narreme (2), building blocks, tangieren die Ebene der histoire. Die SPIEGEL-Webseite erfüllt zunächst die inhaltlichen Basiskriterien, die Festlegung von Zeit und Ort des Geschehens. Dies erfolgt gleich mehrfach: zum einen durch die sichtbare graphische Gestaltung der Seite, durch die aufrufbaren Bilder und Videosequenzen und letztlich durch die Tonmitschnitte - sieht man von den schriftlichen Erläuterungen ab. Zum anderen verweisen die Nennungen «11. September 2001» und «9/11» - als Datumsangaben selbst zu Symbolen verdichtet - ohnehin auf den Ort, der explizit nicht mehr genannt werden muss. Ein drittes Narrem stellen Menschen als narrative Figuren dar, die die Fähigkeit zum SprechHandeln, Denken, sowie physischen und psychischen Leid besitzen ${ }^{3}$ (Wolf 2002, S. 45), auf SPIEGEL-Online realisiert in den fotografischen Porträts der namentlich genannten Personen und in ihren Stimmen, ausgewählt aus den Unterlagen des Gerichts in Alexandria, Virginia. Zwischen diesen drei Elementen entfaltet sich der eigentliche Kern des Narrativen, die Handlung bzw. das Geschehen: Beginnend mit dem ersten Notruf aus einem Flugzeug, dem anschliessenden Einschlag des ersten Flugzeugs in den Nordturm, den Funkmitschnitten der Einsatzkräfte, den Notrufen der Eingeschlossenen, dem Einsturz der Türme und zuletzt den AmateurAufnahmen der Staub- und Aschewolken.

Zu diesen der historie zuzurechnenden Narremen treten aus den Bereichen Schauplatz, Figuren und Handlung (3) Narreme der discourse-Ebene hinzu: Sie betreffen die Syntax, denn «nur so lassen sich die Vorstellungsinhalte, die durch die Bausteine [building blocks, Anm. d. V.] hervorgerufen werden, zu einer dem Schema des Narrativen entsprechenden [...] Geschichte bzw. einem Plot zusammenschliessen» (Wolf 2002, S. 47). Zu diesem Narrem-Komplex zählt allen voran die Chronologie als basales narratives Verknüpfungsprinzip. Die Chronologie gliedert die inhaltlichen Bausteine in einen Ablauf ein und garantiert damit eine spezifische Anordnung der Elemente, beispielsweise durch einen linearen Zeitvektor. Auf der SPIEGEL-Webseite wird die Möglichkeit des chronologischen Betrachtens - wie eingangs erwähnt - auf der Startoberfläche angeboten. Die einzelnen aufrufbaren Fenster, die die building blocks der Narration bilden, sind zeitlich exakt verortet und beginnen mit dem Notruf der Flugbegleiterin um 8.20 und enden mit den Bildern der Aschewolke nach dem Einsturz des zweiten Turms um 10.28. Nach Wolf wird die Chronologie des Geschehens verstärkt, wenn zeitlich bedingt Neues

3 Wolf nennt diese Kategorie inhaltlicher Hohlformen «anthropomorphe Wesen», denn diese Funktion können beispielsweise in schriftlichen Fabeln, aber auch auf Zeichnungen und Bildern Pflanzen oder Tiere einnehmen (Wolf 2002, S. 45). 
mit Altem kombiniert wird, so dass eine Wiederholung eintritt (in der schriftlichen oder mündlichen Erzählung beispielsweise rekurrente Auftritte bzw. Nennungen derselben Personen, vgl. Wolf 2002, S. 47f.). Diese Wiederholung erfolgt auf der SPIEGEL-Webseite meines Erachtens durch den mehrfachen Einbau der Bilder des Einschlags, der Explosion im Südturm und des Einsturzes der Tower. Die Wiederholung erfolgt also auf der visuellen Ebene - ich werde auf diesen entscheidenden Umstand zurückkommen, denn damit ist ein zentraler Effekt des Bildlichen verbunden. Die dritte Narrem-Ebene bildet das Kennzeichen Teleologie: Neben der Chronologie und Wiederholung ist die Ausrichtung auf ein Ziel ein Erkennungsmerkmal von Erzählungen. Dieses «Ende» der Erzählung ist im vorliegenden Beispiel die bildliche Wiedergabe der sich ausbreitenden Aschewolke.

Die Analyse der Webseite mit dem erzähltheoretischen Werkzeug des NarremAnsatzes weist deutlich auf die Tiefenstruktur des Dargestellten hin: Auswahl und Anordnung der Diskursfragmente zeigen eine Struktur der Erzählung. In dieser Erzählung mit dem Titel «Was am Tag des Terrors geschah: 9/11 in Bildern und Tönen» als globital memory field ist es nicht das Medium der Sprache, das die Narration trägt, sondern die Anordnung der Videosequenzen und Fotografien. Es handelt sich um eine Bilder-Geschichte, die sich aus visuellen Narremen zusammensetzt. Im Folgenden möchte ich diese visuellen Narreme (als building blocks auf der Ebene des Bildlichen) in den Mittelpunkt der Auseinandersetzung rücken - beginnend wiederum mit einer These.

\section{Medienikonen und emotionale Erinnerung}

In einem gewissen Sinne handelt es sich - um mit Walter Benjamin zu sprechen - um eine Ästhetisierung des Politischen (Benjamin 2007, S. 48; vgl. zu dieser These in einem anderen Zusammenhang des Schlagworts vom War on Terror Mitterhofer u. Hey-Ehrl 2012, S. 53). In diesem Kontext ist damit der Zusammenhang von (Bilder-)Politik, Ästhetisierung und Emotionalisierung gemeint (vgl. Kirschenmann 2006, S. 140ff.). Pressefotografien von historisch-politischen Ereignissen sind Teil eines «Prozesses der Herausbildung, der Bestimmung und Ausformung von Öffentlichkeit» (Diers 1997, S. 12), sie sind, mit anderen Worten, konstitutiv für Gesellschaften. Auf die dezidiert bildpolitische Dimension der Pressefotografie weist auch Bernd Stiegler mit Blick auf «9/11» hin (Stiegler 2010, S. 226). In diesem Kontext ist insbesondere der Essay Diplopie von Clément Chéroux zu nennen, der die Pressefotografien der Titelseiten von 400 amerikanischen Tageszeitungen am Tag nach den Anschlägen einer Analyse unterzog. Sein Thema ist zum einen die unmittelbare Medialisierung des Ereignisses (Chéroux 2011, S. 12) und die Auswahl der Bilder mit der Kernfrage, «was wir am 11. September gesehen ha- 
ben» (Chéroux 2011, S. 12). ${ }^{4}$ Zum anderen steht die Frage nach den inhaltlichen, historischen Bezügen im Mittelpunkt, genauer, es geht um die Analyse der in den Pressefotografien generierten historischen Analogien (Link 1991), die wiederum eine Rechtfertigung für aktuelles politisches Handeln liefern (Chéroux 2011, S. $60 \mathrm{ff}$.$) .$

Als Fotografien sind Pressefotografien jedoch ästhetisch organisiert, das heisst, die Fotografie ist ein den Regeln und "Gesetzen der Ästhetik verpflichtetes Bild» (Diers 1997, S.23). Das meint zum einen, dass die Pressefotografie nicht nur Zeichen, sondern auch Symbole miteinschliesst, die kollektiv rezipierbar und damit identitätsstiftend sind (Link 1982, S. 13f.); der Gedanke meint zum anderen, dass sich das Politische durch diese Ausrichtung an Ästhetik auch nach ästhetischen Kriterien inszeniert bzw. arrangiert. Zwei Konsequenzen können daraus gezogen werden: (1) In dem Masse wie Pressefotografie «institutionalisiert» (Diers 1997, S. 23) wird, ihr gesellschaftlich legitimierte "Abbild-Hoheit» zukommt («So ist es gewesen.»), greift sie auf bestimmte Weise politische Ereignisse auf - und diesen manchmal «vor». Wie ist dieser letzte Gedanke, der sich auf den amerikanischen Medienwissenschaftler Richard Grusin stützt, zu verstehen? Medien - und damit auch Fotografien - etablieren mediale frameworks (Erll 2007, S. 30f.), also eine Form von Darstellungsmatrix innerhalb derer kommende Ereignisse repräsentiert und gedeutet werden können. Mit anderen Worten und auf die Thematik hier bezogen: Pressefotografie als gesellschaftliche Institution schafft diskursive Darstellungsrahmen für das mediale Festhalten von Ereignissen - zum Beispiel bestimmte visuelle Topoi oder Szenarien, jedenfalls formale Rahmungen. Diese Prozesse nennt Grusin Prämediation im Unterschied zu Remediation; Letzteres meint das bekannte Phänomen, dass bestimmte Medien in anderen wieder auftauchen bzw. reinszeniert werden. ${ }^{5}$ Unter dem Eindruck der Anschläge auf das World Trade Center bedeutet nun Prämediation die Antizipation der Zukunft durch imaginäre Szenarien von Seiten der Medien (beispielsweise in Fernsehsendungen), mit dem Ziel, die Ängste emotional unter Kontrolle zu bringen, wie sie seit 9/11 in der amerikanischen Gesellschaft bestehen (Grusin 2010; Assmann 2012, S. 25f.). Die zweite Konsequenz (2) betrifft das Dargestellte selbst, insbesondere mit Blick auf abgebildete Menschen, die Gestenkommunikation: «Denn Gesten «sprechen» auch im Bild, sie verhelfen dazu, die stummen Bilder beredt zu machen» (Diers 1997, S. 23). Für diese Bedeutung der Gestenkommunikation in Bildern, ihrer Funktion des «Wiedererkennens» von Gesten über Jahrhunderte, prägte der Kunsthistoriker Aby Warburg

4 Der Autor hat zudem eine Bildtypenanalyse erstellt, die das Dargestellte im Prinzip auf relativ wenige Inhalte reduziert, die auch als narrative Sequenzen zu verstehen sind (vgl. Chéroux 2011, S.22 f.).

5 Astrid Erll verweist auf die gängige Praxis, wonach beispielsweise in Computerspielen bzw. -graphiken bekannte Hollywood-Filme re-inszeniert werden und filmische Verfahren inkorporiert werden (vgl. Erll 2007, S. 29). 
den Begriff der «Pathosformel» (Warburg 1979, S. 126). Es handelt sich um aus der Antike übernommene Motive einer Gesten- und Gebärdensprache, die sowohl äussere Bewegung wie innere Bewegtheit (Pathos und Leid) zum Ausdruck bringt. Politik ist dann «erfolgreich» - als Zwischenfazit formuliert - wenn sie, wie Michael Diers es formuliert, "ästhetisch gelingt und bildwürdig erscheint» (1997, S. 24). Durch Arrangement, Montage, Schnitt und Standbild, kurz, durch die nachträglichen, digitalen Bearbeitungsmöglichkeiten des visuellen Materials nach bestimmten, ästhetischen und narrativen Kriterien unterliegen die Bilder, Sequenzen und Fotografien auf der SPIEGEL-Webseite einem bestimmten Mass an Ästhetisierung. Diese Ästhetisierung ist - neben weiteren Faktoren - Bedingung dafür, dass sich bestimmte Pressefotografien zu lkonen verdichten: Die Bilder der brennenden und einstürzenden Türme sind zweifelsfrei die dominanten Medienikonen (zum Begriff: Fahlenbach u. Viehoff 2005, S. 359) des ersten Jahrzehnts.

Ikonen verbanden zu Zeiten ihres kultischen Gebrauchs etwas Abgebildetes, das imago, mit einer historia, einer (Bilder-)Geschichte in Form der Verbindung der Darstellung eines/einer Heiligen mit der entsprechenden Bibelstelle (Fahlenbach u. Viehoff 2005, S. 359). In diesem Sinne verknüpft die Medienikone der SPIEGELWebseite die vermeintlich getreue Abbildung eines historischen Ereignisses - das imago der brennenden, einstürzenden Türme - mit der historia, der (Bilder-)Geschichte des Vormittags des 11. Septembers 2001 in New York. Eine entscheidende Eigenschaft von Medienikonen ist ihre Fähigkeit, emotionale Erinnerungen bei den Betrachtern/-innen hervorzurufen (Kirschenmann 2006, S. 140): Dies betrifft nicht nur die Erinnerung an das historische Ereignis selbst, sondern auch die biographische Situation, in der die Bilder zuerst wahrgenommen wurden (Fahlenbach/ Viehoff 2005, S. 361). Die affektgenerierende Eigenschaft von Medienikonen bildet eine Schnittmenge zu so genannten «Schlagbildern» im Sinne Warburgs. Ein Begriff, den er bereits 1919 in seiner Arbeit «Heidnisch-antike Weissagung in Wort und Bild zu Luthers Zeiten» (Warburg 1979, S. 232) in die Diskussion einbringt. Warburg entwickelt diesen Begriff während des Ersten Weltkriegs anhand der damaligen Presseberichterstattung in Anlehnung an Ausdrücke wie «Schlagwort» und «Schlagzeile» - der Gegenwartsbezug in Warburgs Aufsatz bleibt allerdings implizit (Diers 1997, S. 29). Warburg beschreibt mit diesem Neologismus eine bestimmte Art von Bildern, «verdichtete Bilder», mit der Funktion, eine «ubiquitäre, ganz auf Wirkung verlegte, eindrücklichen Darstellung» (Diers 1997, S. 7) zu evozieren. Einige Charakteristika von Schlagworten gelten auch für Schlagbilder, insbesondere Verkürzung, Wiederholungszwang und Emotionalisierung (Wülfing 1980, S. 33ff.), wobei ich mit Blick auf die Übertragbarkeit der begrifflichen Attribute für die ersten beiden Charakteristika die Begriffe Verdichtung und Wiederholung vorschlagen würde - letzteres demonstriert die Webseite eindrücklich: Es liegt bei den Betrachtern/-innen, wie oft sie sich die Bilder und Videosequenzen ansehen 
möchten. ${ }^{6}$ Entscheidend ist das dritte Charakteristikum eines Schlagwortes: «Wer Schlagworte benutzt, will damit bei dem, den er anspricht, nicht Reflexion auslösen, sondern Emotion» (Wülfing 1980, S. 33). Obwohl beide Konzepte, Schlagbild wie Medienikone, den Aspekt der Emotionalisierung besonders hervorheben, unterscheiden sich Medienikonen von Schlagbildern oder «Schlüsselbildern» (Ludes 2001, S. 51 ff., Paul 2009, S. 29), denn die beiden letzten können zwar «kollektive Erinnerungen an ein konkretes Ereignis bündeln und symbolisch verdichten, deren Semantik [geht] aber nur geringfügig über die Symbolisierung und Veranschaulichung eines konkreten Ereignisses hinaus» (Fahlenbach u. Viehoff 2005, S. 362). Anders bei Medienikonen: hier könnte von einem Symbolisierungs-«Überschuss» gesprochen werden.

Es ist meines Erachtens gerade dieser spezifische Symbolisierungs-«Überschuss» medialer Ikonen, der als Grundbedingung einer Ästhetisierung des politischen Ereignisses unverzichtbar ist. Ikonisierung stellt auf diese Weise eine zugespitzte und gebündelte Visualisierung dar: Ein Ereignis tritt besonders deutlich und nachhaltig in Erscheinung (Leggewie 2009, S. 9). Diese «lkonabilität» (Paul 2009 zitiert nach Leggewie 2009, S.9) ist eine Verschmelzung von historischer Signifikanz, ästhetischen Bildeigenschaften und (massen-)medialer, wiederholender Reproduktion. Damit sind zumindest drei wesentliche Eigenschaften (vgl. Leggewie 2009, S. 9f.) von Medienikonen verknüpft: (1) Sie mobilisieren kollektive Affekte, denn sie verdichten Emotionen wie Ängste, Wut und Trauer zu Symbolen menschlichen Daseins und Leidens (Czech 2006, S. 27). (2) Als «Ereignisikonen» (Paul 2009, S. 29) - mit ihrer spezifischen Ästhetik (Kirschenmann 2006, S. 141) - erregen sie besondere öffentliche Aufmerksamkeit. Die Verknüpfung eines herausragenden historischen Ereignisses mit bedeutenden Persönlichkeiten oder erschütternden Einzelschicksalen betroffener Individuen ist meist umgeben von einer «legendären Aura aus Gerüchten, Anekdoten und Ruhm» (Czech 2006, S. 27), also von Verschwörungstheorien oder von Bildern der Täter, Opfer und Helden des 11. September. (3) Medienikonen modellieren kollektive und individuelle Erinnerung, ${ }^{7}$ insbesondere - wie genannt - emotionale Erinnerung. Dazu bedarf es allerdings einer spezifischen medialen «Technik»: der permanenten Wiederholung in Gestalt von Re-Inszenierungen. Mittels wiederholender Re-Inszenierung ist ein hohes

6 Im Kontext der medialen Berichterstattung zu «9/11» hat das Phänomen der Wiederholung von Beginn an und mit Blick auf die spätere Ikonisierung eine enorme Bedeutung: Dies zeigt Chéroux in seinem Essay Diplopie zum einen für den Fernsehnachrichtenbereich (Chéroux 2011, S. 16) wie für die von ihm genauer untersuchten 400 Titelseiten amerikanischer Tageszeitungen (Chéroux 2011, S. 17 f.).

7 An dieser Stelle sei ein Beispiel genannt: Im Zusammenhang mit den Terroranschlägen vom 11. September sind inzwischen einige Arbeiten erschienen, die sich mit dem berühmten Bild der Flagge hissenden Feuerwehrmänner auf den Trümmern des World Trade Centers am 11. September 2001 und seines historischen Bild-Bezugs auseinandersetzen: der Erstürmung der Pazifikinsel Iwojima 1945 durch amerikanische Soldaten, «festgehalten» (nachinszeniert) vom Kriegsberichterstatter und Fotografen Joe Rosenthal (stellvertretend Chéroux 2011, S. 60 ff.). 
Mass an Wiedererkennungswert - zu denken ist an das Narrem der Wiederholung - garantiert: Exakt durch diesen Prozess verknüpft sich die Vertrautheit der Bilder (Wer kennt sie nicht?) mit einem dauerhaften und stets aufs Neue eintretenden Schrecken - den die SPIEGEL-Webseite verspricht - bei der Betrachtung der Bilder. Diese Verflechtung von historischem Ereignis und Emotion zeigt zumindest zwei Effekte: Einerseits nehmen Bilder dieser "Qualität» eine zunehmend politische und weltanschauliche Funktion an; sie «synchronisieren» Bevölkerungen, um einen Begriff von Claus Leggewie aufzugreifen (2009, S. 10). Aus einer anderen philosophischen Richtung gedacht, sehe ich im re-inszenierten Wiedererkennen eine ideologische Anrufung im Sinne Louis Althussers (2010, S. 88). Andererseits werden in der Re-Inszenierung Werte etabliert, die dauerhaft im kollektiven Gedächtnis und im Habitus der Mitglieder von Gesellschaften verankert bleiben (Fahlenbach u. Viehoff 2005, S. 361).

Es ist Kathrin Fahlenbach und Reinhold Viehoff in ihrer Auseinandersetzung mit historischen Prozessen der Ikonisierung von Bildern zuzustimmen, dass neben den genannten Effekten ein grundlegender Faktor berücksichtigt werden muss: Die Bedeutung der Auswahl und Re-inszenierung der Bilder durch historische Akteure/-innen, Bildproduzenten/-innen und Bildvermittlern/-innen ${ }^{8}$, die die Bilder als «Identifikationsflächen» (Fahlenbach u. Viehoff 2005, S. 362) bzw. «Applikationsvorlagen» (Link 1982, S. 13f.) für die breite Aufnahme und Rezeption «anbieten». Diese gestalterische und semantische Kodierung (Fahlenbach u. Viehoff 2005, S. 362) stellt so etwas wie eine Rahmung, den Darstellungsrahmen im Prozess der Ikonisierung, her. Dieser Rahmung möchte ich im Anschluss an George Didi-Huberman den Namen «Repräsentationsdispositiv» geben (Didi-Huberman 2009, S. 52). Didi-Huberman führt den Begriff nicht aus, stützt sich aber - wie seine Argumentation im Text zu interpretieren ist - an dieser Stelle auf Michel Foucault: Foucault versteht mit diesem Begriff das In-Beziehung-Setzen bzw. das In-Beziehung-Sein heterogener Elemente, im Kontext hier zunächst von Diskursfragmenten aus verschiedenen digitalen Archiven. Das Repräsentationsdispositiv ist als Rahmung zu sehen, das Institutionen wie Verlage, Pressehäuser und die darin agierenden Akteure/-innen in Beziehung setzt. Das Repräsentationsdispositiv selbst ist nichts eigentlich Materielles (vgl. Foucault 1978, 119f.), sondern das Netz, das zwischen diesen Elementen geknüpft werden kann. Die Verknüpfung auf der SPIEGEL-Webseite erfolgt im Modus der Narration mittels visueller Narreme als building blocks mit der Funktion - und das ist entscheidend - «Bilder in Umlauf zu bringen» (Didi-Huberman 2009, S. 51). Diese Bilder stehen repräsentativ für bestimmte (historische) Ereignisse. Durch dieses In-Beziehung-Setzen innerhalb

8 Zur Frage der Rolle von Presseagenturen, allen voran Associated Press, der Akteure/-innen in einem globalen Bildermarkt, Prozessen der Homogenisierung und Hegemonialisierung durch Nachrichtenagenturen vgl. für die ersten Tage nach den Attentaten auf das World Trade Center Chéroux 2011, S. $47 \mathrm{ff}$. 
eines Repräsentationsdispositivs ist der institutionelle Rahmen berücksichtigt, der Prozesse der Ikonisierung überhaupt erst ermöglicht.

Es stellt sich nun die Frage, welchen Effekt diese Prozesse der Ikonisierung - insbesondere im Kontext von Ereignisikonen wie den Bildern der Anschläge auf das World Trade Center - hervorbringen können. Das Bild des Ereignisses erscheint als Abbild der Geschichte. Auf diese Weise - und das als schliessende These kann es als Fetisch im Sinne von Karl Marx zu fungieren beginnen. ${ }^{9}$ Mit dieser Aussage soll keinem neuen Ikonoklasmus das Wort geredet werden, ganz im Gegenteil: Es ist nicht das Bild an sich, sondern sein Funktionieren innerhalb des Repräsentationsdispositivs. Marx argumentiert im Kontext des Warentausches, dass die gesellschaftliche Bedingtheit der Ware im Tauschprozess verkannt wird. Die Ware erscheint nicht als gesellschaftliche Form, ihr Wert wird ihr als Naturtatsache zugesprochen (Marx 1988, S $88 \mathrm{ff}$.). Die gesellschaftlichen Strukturen im Produktionsprozess und im Tausch bleiben verborgen. In diesem Sinne lässt sich sagen: Wenn sich die gesellschaftliche Bedingtheit der Entstehung der Bilder, der Bedingungen ihrer Distribution und Konsumption von ihnen selbst löst, wird das historische Bild wie in der konkreten Inszenierung auf SPIEGEL-Online eigentümlich a-historisch: In der Inszenierung, in der Reproduktion der Bilder vom Ereignis, suggeriert das Bild die getreue Wiedergabe der Realität, aber nicht auf der Ebene ihres gesellschaftlichen Zustandekommens, sondern als ein ausserhalb der Subjekte sich ereignendes Ereignis und damit quasi schicksalshaft ("So ist es gewesen. Das zeigen die Bilder.»). Damit werden die Bilder auf der SPIEGEL-Seite zu einem visuellen Fetisch einer überzeitlichen Katastrophe, eines von aussen hereinbrechenden Schicksals. Dazu müssen sie, wie zu zeigen war, in eine spezifische Struktur eingebettet sein: in die Erzählform - hier in die Gattung des bildhaften Melodrams, der visualisierten Tragödie. In Analogie zur Warenzirkulation bei Marx und dem Fetisch-Charakter der Ware lässt sich formulieren: Das Repräsentationsdispositiv bringt tatsächlich «die Bilder in Umlauf». Nimmt man die Analogie zum Tauschwert der Ware und seiner allgemeinen Äquivalentform, dem «Geld», dann ist Leggewie zuzustimmen, dass Bilder in dieser Funktionsweise zu einer «Währung visueller Massenkommunikation» werden (Leggewie 2009, S. 10). Was wird in diesem Tauschvorgang verkannt? - Die Möglichkeit der Erkenntnis der realen, historisch-politischen Verhältnisse, die die Bilder hervor- und in «Umlauf bringen». Diese gesellschaftlichen Verhältnisse, angeordnet in einem Repräsentationsdispositiv, sind - für die Formung visueller Kompetenz - meines Erachtens immer zu berücksichtigen.

9 Zur folgenden Argumentation vgl. Ebbrecht im Zusammenhang mit dem Deutschland-Bild, der 2008 einen Eröffnungsvortrag unter dem Titel «Die Liebe zum Bild. Nostalgie, Fetisch, Dialektik: Das Bild in der Erinnerungskultur» hielt. http://www.extrablatt-online.net/archiv/ausgabe-4/tobiasebbrecht-die-liebe-zum-bild.html. 


\section{Literatur}

Assmann, Aleida. 2012. "Resonanz und Einschlag: Zur Affektlogik von Bildern im kulturellen Gedächtnis.» In Bild und Bildlichkeit, hrsg. v. Otfried Höffe, 23-35. Nova Acta Leopoldina Nummer 386, Band 113. Stuttgart: Wissenschaftliche Verlagsgesellschaft.

Assmann, Jan. 2007. Das kulturelle Gedächtnis: Schrift, Erinnerung und politische Identität in frühen Hochkulturen. 6. Aufl. München: Beck.

Benjamin, Walter. 2007. Das Kunstwerk im Zeitalter seiner technischen Reproduzierbarkeit und weitere Dokumente. Kommentar von Detlev Schöttker. 1. Aufl. Frankfurt am Main: Suhrkamp.

Chéroux, Clément. 2011. Diplopie: Bildpolitik des 11. September. Konstanz: University Press.

Czech, Alfred. 2006. "Bildkanon im Spannungsverhältnis zwischen individuellem und kollektivem Bildgedächtnis.» In Bilder, die die Welt bedeuten: «lkonen» des Bildgedächtnisses und ihre Vermittlung über Datenbanken, hrsg. v. Johannes Kirschenmann u. Ernst Wagner, 9-40. München: kopaed.

Didi-Huberman, Georges. 2009. «Klagebilder, beklagenswerte Bilder?» Zeitschrift für Medienwissenschaft 1 (1): 50-60.

Diers, Michael. 1997. Schlagbilder: Zur politischen Ikonographie der Gegenwart. Frankfurt am Main: Fischer Taschenbuch Verlag.

Erll, Astrid. 2007. Prämediation - Remediation. Repräsentation des indischen Aufstands in imperialen und post-kolonialen Medienkulturen (von 1857 bis zur Gegenwart). Trier: Wissenschaftlicher Verlag.

Fahlenbach, Kathrin und Reinhold Viehoff. 2005. «Medienikonen des Krieges: Die symbolische Entthronung Saddams als Versuch strategischer Ikonisierung.» In War Visions: Bildkommunikation und Krieg, hrsg. v. Thomas Knieper u. Marion G. Müller, 356-387. Köln: Herbert von Halem Verlag.

Foucault, Michel. 1978. «Ein Spiel um die Psychoanalyse. Gespräch mit Angehörigen des Departement de Psychoanalyse der Universität Paris/Vincennes.»In Dispositive der Macht: Michel Foucault über Sexualität, Wissen und Wahrheit, 118-175. Berlin: Merve Verlag.

Foucault, Michel. 1990. Archäologie des Wissens. Frankfurt am Main: Suhrkamp.

Grusin, Richard A. 2010. Premediation: Affect and mediality after 9/11. Basingstoke, New York: Palgrave Macmillan.

Jäger, Siefried. 2004. Kritische Diskursanalyse: Eine Einführung. 4. Aufl., Münster: Unrast Verlag.

Kirschenmann, Johannes. 2006. «Voller Emotionen und Erinnerungen»-Das kollektive Gedächtnis und seine mediale Konstruktion.» In Bilder, die die Welt bedeuten. «lkonen» des Bildgedächtnisses und ihre Vermittlung über Daten- 
banken, hrsg. v. Johannes Kirschenmann u. Ernst Wagner, 135-149. München: kopaed.

Leggewie, Claus. 2009. «Zur Einleitung: Von der Visualisierung zur Virtualisierung des Erinnerns.» In Erinnerungskultur 2.0: Kommemorative Kommunikation in digitalen Medien, hrsg. v. Erik Meyer, 9-28. Frankfurt am Main u. New York: Campus-Verlag.

Link, Jürgen. 1982. "Kollektivsymbolik und Mediendiskurse: Zur aktuellen Frage, wie subjektive Aufrüstung funktioniert.» kultuRRevolution: Zeitschrift für angewandte Diskurstheorie 1 (1): 6-21.

Link, Jürgen. 1991. «Historische Analogien: Strukturen und Funktionen.» kultuRRevolution: Zeitschrift für angewandte Diskurstheorie 24: 3-9.

Ludes, Peter. 2001. Multimedia und Multi-Moderne: Schlüsselbilder. Fernsehnachrichten und World Wide Web - Medienzivilisierung in der Europäischen Währungsunion. 1. Aufl. Wiesbaden: Westdeutscher Verlag.

Marx, Karl. 1988. Das Kapital: Kritik der politischen Ökonomie. MEW, Band 23., 17. Aufl. Berlin: Dietz Verlag.

Meyer, Erik. 2009a. «Vorwort.»In Erinnerungskultur 2.0: Kommemorative Kommunikation in digitalen Medien, hrsg. v. Erik Meyer, 7. Frankfurt am Main u. New York: Campus-Verlag.

Meyer, Erik. 2009b. «Erinnerungskultur 2.0? Zur Transformation kommemorativer Kommunikation in digitalen, interaktiven Medien.» In Erinnerungskultur 2.0: Kommemorative Kommunikation in digitalen Medien, hrsg. v. Erik Meyer, 175206. Frankfurt am Main u. New York: Campus-Verlag.

Mitterhofer, Hermann und Ekkehard Hey-Ehrl. 2012. "Das Bild des traumatisierten Körpers: Von der visuellen Narration des Leidens anderer.» Zeitschrift für Psychotraumatologie, Psychotherapiewissenschaft und Psychologische Medizin 10 (2): 47-59.

Paul, Gerhard. 2009. «Das Jahrhundert der Bilder: Die visuelle Geschichte und der Bilderkanon des kulturellen Gedächtnisses.» In Das Jahrhundert der Bilder. 1900 bis 1949, hrsg. v. Gerhard Paul, 14-39. Göttingen: Vandenhoeck \& Ruprecht.

Reading, Anna. 2011. "Memory and Digital Media: Six Dynamics of the Globital Memory Field.» In On Media Memory: Collective Memory in a New Media Age, hrsg. v. Motti Neiger, Oren Meyers u. Eyal Zandberg, 241-252. Houndmills, Basingstoke, Hampshire, New York: Palgrave.

Stiegler, Bernd. 2010. «Einleitung zu Fotografie und Gesellschaft.» In Texte zur Theorie der Fotografie, hrsg. v. Bernd Stiegler, 225-229. Stuttgart: Philipp Reclam jun. 
Warburg, Aby. 1979. «Dürer und die italienische Antike.» In Aby Warburg: Ausgewählte Schriften und Würdigungen, hrsg. v. Dieter Wuttke, 1. Aufl., 125-130. Baden-Baden: Verlag Koerner.

Warburg, Aby. 1979. «Heidnisch-antike Weissagung in Wort und Bild zu Luthers Zeiten. Sitzungsberichte der Heidelberger Akademie der Wissenschaften 1919.» In Aby Warburg: Ausgewählte Schriften und Würdigungen, hrsg. v. Dieter Wuttke, 1. Aufl., 199-304. Baden-Baden: Verlag Koerner.

Wolf, Werner. 2002. «Das Problem der Narrativität in Literatur, bildender Kunst und Musik: Ein Beitrag zu einer intermedialen Erzähltheorie.» In Erzähltheorie transgenerisch, intermedial, interdisziplinär, hrsg. v. Vera Nünning und Ansgar Nünning, 23-104. Trier: WVT Wissenschaftlicher Verlag.

Wülfing, Wulf. 1982. Schlagworte des Jungen Deutschland. Mit einer Einführung in die Schlagwortforschung. Berlin: Erich Schmidt Verlag.

Internetquellen

http://www.spiegel.de/flash/0,5532,26783,00.html

http://www.spiegel.de/panorama/justiz/moussaoui-beweise-online-terrorpuzzlemit-1200-teilen-a-429858.html

http://www.extrablatt-online.net/archiv/ausgabe-4/tobias-ebbrecht-die-liebezum-bild.html 